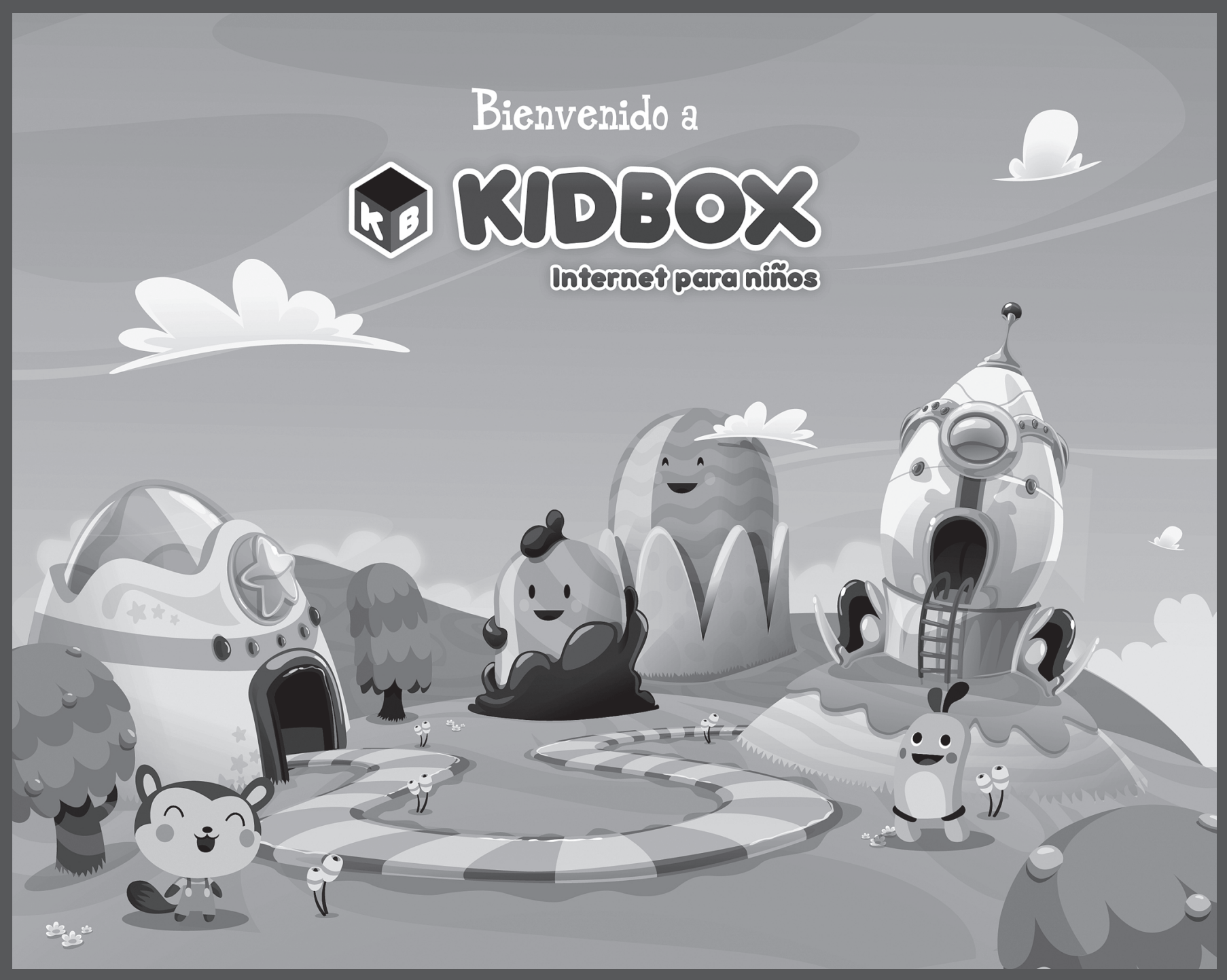

Imagen de bienvenida a Kidbox que aparece al abrir el programa. 


\title{
Kidbox, un mundo feliz en Internet
}

\author{
Por Betina Gersberg
}

\section{El origen}

En un almuerzo familiar de sábado, Alan Kind, ingeniero en computación interesado en aplicaciones web, y Martin Larre, abogado con experiencia en la venta de productos editoriales infantiles, conversaban sobre la problemática aún no resuelta en torno al vínculo de los niños e Internet. Notaron que los padres querían usar la red de la mejor manera, pero que esa intención era relegada por el miedo que le tenían. Entonces, con esos dos pilares como objetivos, la de maximizar la experiencia del medio y la de experimentarlo con seguridad, surgió la idea de crear una solución que diera respuesta a esas dos necesidades. Es así que, además de cuñados, estos profesionales se convirtieron en socios fundadores de Kidbox.

\section{El producto y el servicio}

"La diferenciación entre producto y servicio tiene una línea muy fina. Kidbox es un servicio, porque está a disposición para resolver un problema real", afirma Kind. Lo que ofrecen es una solución para familias con niños de 2 a 8 años, del mercado de habla hispana y portuguesa, aunque los emprendedores tienen proyectada la internacionalización a otros países y a otros idiomas. El eje de Kidbox es un software que permite al niño explorar el mundo del ciberespacio bajo un ambiente seguro, a través de un navegador que presenta contenidos educativos y de entretenimiento infantil. Estos contenidos son seleccionados, categorizados y actualizados por especialistas y se muestran de manera personalizada, adaptados al perfil de cada usuario. El programa, que se descarga gratuitamente de Internet, se ejecuta a pantalla completa y solo se puede salir con una contraseña elegida por el padre. De esta forma el niño no puede acceder a los navegadores tradicionales y se evita su exposición a sitios inapropiados y a personas inapropiadas, contactadas por chats o redes sociales.

Un beneficio adicional es que, al mantener al niño dentro del mundo Kidbox, se evita el daño de archivos locales en las computadoras. A su vez, se ofrece un panel de control en el que los padres pueden conocer las actividades del niño y gestionar los contenidos. En este sentido, Kind asocia la experiencia del navegador infantil con sus recuerdos de las vacaciones de verano: "Tus padres te monitoreaban pero también se adaptaban a lo que disfrutabas hacer. Nosotros queremos pasar esa misma experiencia a lo virtual: que el padre esté al tanto de lo que hace su hijo, pero también que lo deje libre a su imaginación para que explore lo que más le guste".

\section{La experiencia de personalización}

"Antes de Kidbox, si un padre quería proteger a su hijo en Internet tenía que utilizar los típicos filtros o controles parentales de los sistemas operativos. Pero este procedimiento tenía sus desventajas, porque los niños solo accedían a los contenidos de los sitios que sus padres agregaban al filtro. Esto requería a los adultos un permanente trabajo de actualización y, por
Ficha técnica

Producto:: Kidbox, servicio web de navegación segura para niños

Año de publicación online:: noviembre 2011

País:: Uruguay

Responsables:: Alan Kind (uruguayo) y Martín Larre (argentino)

Sitio web::

"www.kidbox.net" 
otra parte, los niños se sentían restringidos en las posibilidades de exploración. Entonces no había una solución como la que nosotros queríamos ofrecer", explica Kind.

Durante el desarrollo de su idea, Kind y Larre descubrieron que existía un servicio similar en Estados Unidos aunque con una gran diferencia: la experiencia de personalización que ofrece Kidbox. Esta permite que, por ejemplo, un niño de cuatro años argentino y una niña de siete de México reciban contenidos diferentes según su perfil. Inicialmente definida por edad, nacionalidad y género, la personalización se extiende a medida que van aprendiendo más de cada usuario; se basa en sus intereses personales, en los de otros niños con un perfil similar y también en quienes presentan un perfil distinto, pero que estuvieron experimentando los mismos contenidos y, por lo tanto, pueden captar la atención.

\section{Un premiado proceso de concreción}

Definida la idea inicial, ambos emprendedores comenzaron a trabajar, con la ayuda externa de referentes locales, sobre un plan de negocios que luego presentaron a la Agencia Nacional de Investigación e Innovación (ANII), de la que consiguieron un primer subsidio para el proyecto. Después generaron un prototipo de manera tercerizada y, más adelante, con el apoyo del Fondo Emprender en Uruguay, formaron el equipo y comenzaron a desarrollar el producto dentro de las oficinas que habitan hoy día, ya con el objetivo de lanzar el servicio.

De los varios premios que obtuvo Kidbox al día de hoy, Kind destaca tres instancias de reconocimiento. Una de ellas fue el Festival of Media LatAm de Miami, en octubre de 2011, en el que ganaron como la compañía innovadora del año porque el jurado consideró que se innovó en torno a la forma en que se establece la comunicación entre padres e hijos. “Ahí validamos el modelo como plataforma de comunicación. Después estuvo el Desafío Intel, en el que quedamos incluidos en el top 10 del mundo y, si bien no ganamos, representó un reconocimiento a nivel tecnológico. Y la tercera instancia fue en la Red Innova en San Pablo, que es una competencia de negocios de startups ${ }^{1}$ en Internet, que ganamos entre 500 empresas. Entonces fue una validación de nuestros pares de lo que estamos haciendo en negocios".

\section{Sortear las dificultades}

"En Uruguay el ecosistema no es fácil para emprender en tecnología. Si hubiéramos tenido acceso a capital, como hay en otros lugares de innovación, seguramente hubiésemos acelerado mucho los tiempos. Nos hubiese gustado lanzar la versión gratuita y tener feedback de los usuarios mucho antes de lo que lo hicimos. Pero en los demás aspectos los tiempos se fueron dando como lo teníamos planeado". Kind explica que en todo el proceso se apoyaron en profesionales específicos para cada área a nivel nacional. En programación trabajaron con una reconocida compañía de desarrollo de software. En contenidos fueron asesorados en un principio por Roberto Balaguer, psicólogo referente en Internet y los niños, y a nivel visual Fernando Picún -quien desarrolló un juego muy descargado del Plan Ceibal-creó el mundo de Kidbox. "O sea, en todas las áreas nos apoyamos en gente que sabe realmente sobre su trabajo. Y fue una sorpresa positiva encontrarnos con la calidad y el talento local en Uruguay".

\section{Mejora continua}

La primera versión gratuita online, que se publicó en noviembre de 2011, es muy diferente a la que se ofrece hoy. "Semana a semana fuimos iterando sobre el 
mismo producto para sacar actualizaciones con mejoras. La definición de algunas funcionalidades, tanto para padres como para chicos, requirió de una profundización mucho mayor de la que teníamos pensada al inicio". Kind lo explica con un ejemplo: "Con la interfaz definida apuntábamos más a niños de 6 a 8 años que de 2 a 5 años, entonces comenzamos a generar una versión, que está aún en proceso, especialmente para niños de esa edad y que se va a diferenciar en una interfaz más simple, con íconos más grandes, sin texto, con un acceso más rápido a la información y un poco más de sonidos que ayuden en la navegación. En realidad la etapa de sondeo no va a terminar nunca, la postura es siempre tratar de mejorar y mejorar".

\section{La forma y el contenido}

Hay dos aspectos fundamentales en Kidbox: el atractivo visual y el contenido, personalizado para el niño. Para crear la estética, Fernando Picún trabajó a partir de unas ilustraciones de sitios web japoneses que, según el equipo, estaban marcando tendencia. "Él dio vida a un mundo súper alegre, muy colorido, en el que no había formas humanas sino personajes, y eso fue evolucionando hasta lo que es hoy, y va a seguir en evolución. Hay que estar en constante cambio, los chicos te piden eso. Si bien el parámetro del mundo de Kidbox ya está establecido, tiene mucho para crecer".

Los contenidos que provee Kidbox a sus usuarios son el resultado de un proceso de búsqueda, selección y categorización a cargo de un equipo de maestras y de una psicóloga que evalúa todos los aspectos referidos a su calidad. "Si en lo visual un contenido no es atractivo o si no satisface sus expectativas, va a ser muy difícil que el niño se enganche. Entonces, por un lado la estética, por otro, los contenidos y una personalización que conjugue ambas cosas”, resume Kind.

\section{Trabajar en equipo}

En esta empresa uruguaya trabajan hoy siete personas dentro de la oficina y cuatro de manera freelance. Sobre la modalidad de trabajo, Kind afirma que en una startup es esencial el trabajo en equipo y la motivación. "Antes de integrar a alguien tratamos de mostrarle la visión que tenemos de Kidbox. Aclaramos que se van a pasar momentos difíciles y muy buenos. En cada etapa compartimos los logros y también las dificultades con las que nos encontramos, las alegrías de un premio, de un reconocimiento o una publicación en un diario o un blog internacional. Eso va generando una comunión entre nosotros, una identificación con lo que vamos haciendo muy importante para no perder el foco y para tener la camiseta puesta y salir adelante. Creo que en una empresa como esta, más que en una compañía que está establecida desde hace años, se necesita mucho del empuje de cada uno y para ello es esencial que exista un marco que lo soporte, y ese marco es el grupo humano que trabaja en conjunto".

\section{Cuanto más simple mejor}

A lo largo de la creación de Kidbox, los fundadores tuvieron que vincularse con distintos públicos para lograr diferentes objetivos. "Como emprendedor tecnológico sos un articulador y tenés que comunicar de diversas maneras en diferentes ámbitos. Una comunicación es hacia adentro, con el equipo humano; otra es la que planteás en tu sitio web o tu Facebook corporativo para los usuarios. Otra forma de transmitir se genera cuando estás negociando contratos. Lo que aprendí es que para tener una buena comunicación hay que saber bien quién es el interlocutor y prepararse para que todo fluya mucho mejor. Sobre lo que comunicás de adentro hacia afuera, con los usuarios, aprendimos que cuanto más simple, mejor. Por ejemplo, el video que ahora está en
Betina Gersberg:: (Montevideo, 1980) es egresada de la Licenciatura en Comunicación Social de la Universidad Católica del Uruguay. Integra el equipo de la Secretaría de Comunicación Institucional de la misma universidad. Se dedica a la comunicación organizacional y audiovisual de manera independiente. Forma parte del equipo de la revista dixit desde su creación, en 2006.

bgersberg@ucu.edu.uy 
la página antes era más complejo y el mensaje más rebuscado. Nos dimos cuenta de que aunque a veces se omitan algunas cosas, cuanto más simple, más efectivo, más claro y más entendible. Y si hay alguna pregunta, el usuario después viene a vos".

\section{Los niños e Internet}

A Kind lo sorprendió la velocidad con la que aprenden los niños. "Nosotros no estábamos preparados para su ritmo y tuvimos que adaptarnos. Por ejemplo, nos dimos cuenta de que las películas tienen un rol preponderante, entonces reajustamos nuestros procesos de actualización de contenidos para seguir captando su atención; ahora proyectamos con un año de antelación qué películas se vienen”. Sobre el manejo del mouse pensaron que iba a ser una barrera. “'¿Cómo van a poder sincronizar la mano y la pantalla?', nos preguntábamos, y en las pruebas nos dimos cuenta de que niños de dos años al toque lograban la sincronización. También aprendimos a diferenciarlos. Por ejemplo, los de 2 a 5 años no se fijan en el orden para llegar a la información, sino en que sea fácil el acceso a ella. En cambio, los de 6 a 8 años empiezan a valorar el camino para llegar a lo que buscan. Y esto influyó directamente en la mejora del servicio". Sobre Internet, el aspecto que más le apasiona a Kind es la globalidad del medio. "Nos pegó de muchas maneras. Desde haber participado en diferentes competencias a nivel mundial y regional, hasta ser publicados en diarios y blogs de todo el mundo. Todo eso se logró simplemente por estar. Tener una cierta visibilidad en algunos premios nos dio una viralidad para que nos bajaran en países como China, Malasia o Rusia, cosas que en un mundo de hace veinte años ni te imaginabas. La globalidad también influyó en que desde el inicio pensáramos Kidbox para Latinoamérica o para Brasil”. De hecho, el proyecto ya obtuvo respuestas de padres que se comunican desde Colombia, EEUU y Guatemala para comentar cómo les ayuda el servicio. Fue justamente este aspecto lo que decidió a Kind a dedicarse a este proyecto: "Sentir que algo sale de tu cabeza y podés influir con un granito de arena a tu alrededor y en el mundo: eso es una gran motivación”.

\section{El futuro}

"La tendencia de los niños a permanecer en Internet va ser cada vez mayor, porque hay cada vez más dispositivos móviles, y por lo tanto la problemática va a aumentar". En este sentido, las expectativas que Kind y Larre tienen a futuro son muchas. "Queremos lanzar cuanto antes la versión premium, que va a prestar más funcionalidades tanto para padres como para niños. Después, la versión en portugués, porque va a ser clave para posicionarnos en Brasil. Me encantaría pensar, para más adelante, en un Kidbox para Japón y para otros países fuera de la región. También estamos pensando en buscar soluciones para niños mayores de 8 años y posicionarnos como una compañía de solución integral para niños en diferentes edades a nivel mundial". Si bien inicialmente la empresa se enfocó en Windows, porque la gran mayoría de las computadoras de la región funcionan con este sistema operativo, están desarrollando complementos para Android y Mac. "Por la forma intuitiva de interactuar con pantallas táctiles, el futuro para niños está en los tablets, entonces vamos a enfocarnos en soluciones para esos dispositivos; ahí nos tenemos que dirigir".:- 